\title{
News and Announcement
}

We are pleased to announce that the Editorial Board Member of NS, Kuo-Chen Chou, has been identified by Science Watch (http://sciencewatch.com/ana/fea/09maraprFea/) as the author with the highest numbers of Hot Papers published over the preceding two years (2007 and 2008). Among the 13 authors listed in the table of "Scientists with Multiple Hot Papers" by Science Watch, Professor Dr. Kuo-Chen Chou of Gordon Life Science institute and Shanghai Jiaotong University ranks No.1 with 17 hot papers.

Meanwhile, the review article by Kuo-Chen Chou and Hong-Bin Shen, entitled "Recent Progresses in Protein Subcellular Location Prediction" published in Analytical Biochemistry, has been identified by Science Watch as the New Hot Paper in the field of Biology \& Biochemistry (http://sciencewatch.com/dr/nhp/2009/09marnhp/09marnhpChou/).

For more information about the hot research and hot papers, go to visit the web-sites at http://www.sciencenet.cn/htmlnews/2009/3/216833.html; http://sciencewatch.com/ana/fea/pdf/09maraprFea.pdf; and http://sciencewatch.com/dr/nhp/2009/pdf/09marnhpChou.pdf.

Please join us to send our sincere and warm congratulations to our fellow board member, Kuo-Chen Chou, for his prominent contributions in science. Meanwhile, we hope this announcement can attract more researchers to submit their best papers to NS, the journal that publishes the highest quality of research and review articles in all important aspects of natural sciences and their intersection. 